Title :

\title{
Oblique Detonation Waves Stabilized in Rectangular-Cross-Section Bent Tubes
}

Authors :

Affiliation 1 :

Affiliation 2 :

Address :

Colloquium :

Total Length :

(Method : M1)
YUSUKE KUDO ${ }^{1}$, YUUTO NAGURA ${ }^{1}$, JIRO KASAHARA ${ }^{1}$

YUYA SASAMOTO $^{2}$, AKIKO MATSUO ${ }^{2}$

Department of Engineering Mechanics and Energy, University of Tsukuba 1-1-1 Tennodai, Tsukuba 305-8573, Japan

Department of Mechanical Engineering, Keio University

3-14-1 Hiyoshi, Kouhoku-ku, Yokohama 223-8522, Japan

JIRO KASAHARA, Department of Engineering Mechanics and Energy,

University of Tsukuba, 1-1-1 Tennodai, Tsukuba 305-8573, Japan

E-mail : kasahara@kz.tsukuba.ac.jp

Fax : 029-853-5267

DETONATIONS, EXPLOSIONS AND SUPERSONIC COMBUSTION

including pulse-detonation and scramjet engines

5981 words

$\begin{array}{lr}\text { Main text : } & 2243 \text { words } \\ \text { Acknowledgements : } & 293 \text { words } \\ \text { References : } & 404 \text { words } \\ \text { Figures : } & 2785 \text { words } \\ \text { Tables : } & 256 \text { words }\end{array}$




\begin{abstract}
Oblique detonation waves, which are generated by a fundamental detonation phenomenon occurring in bent tubes, may be applied to fuel combustion in high-efficiency engines such as a Pulse Detonation Engine (PDE) and a Rotating Detonation Engine (RDE). The present study has experimentally demonstrated that steady-state oblique detonation waves propagated stably through rectangular-cross-section bent tubes by visualizing these waves using a high-speed camera and the shadowgraph method. The oblique detonation waves were stabilized under the conditions of high initial pressure and a large curvature radius of the inside wall of the rectangular-cross-section bent tube. The geometrical shapes of the stabilized oblique detonation waves were calculated, and the results of the calculation were in good agreement with those of our experiment. Moreover, it was experimentally shown that the critical condition under which steady-state oblique detonation waves can stably propagate through the rectangular-cross-section bent tubes was the curvature radius of the inside wall of the rectangular-cross-section bent tube equivalent to 14 to 40 times the cell width.
\end{abstract}

\title{
Keywords
}

Detonation Wave, Oblique Detonation Wave, Pulse Detonation Engine, Rotating Detonation Engine

\section{Nomenclature}

$p_{0} \quad=$ initial pressure

$r_{\mathrm{i}}=$ curvature radius of the inside wall

$V_{\mathrm{CJ}}=$ Chapman-Jouguet $(\mathrm{CJ})$ detonation velocity

$\phi \quad=$ analogy angle

$\lambda=$ detonation cell size

$\theta \quad=$ angle of detonation wave front

$\theta_{\mathrm{i}}=$ angle of detonation wave front on inside wall

$\omega=$ angular velocity of detonation wave front

$\omega_{\mathrm{i}}=$ angular velocity of detonation wave front on inside wall

\section{Introduction}

Oblique detonation waves, which are generated by a fundamental detonation phenomenon in bent tubes, may be applied to fuel combustion in high-efficiency engines such as a Pulse Detonation Engine (PDE) (Kailasanath [1, 2], Roy et al. [3], Kasahara et al. [4-6], Sato et al. [7], Endo et al. [8, 9]) and a Rotating Detonation Engine (RDE) (Meredith et al. [10], Kindracki et al. [11], Jiang et al. [12], Yi et al. [13]). Many studies have been conducted on oblique detonation waves, which are stably generated by flying particles projected together with a combustible premixed gas (Kasahara et al. [14-17], Higgins [18-19], Lee [20], Kaneshige and Shepherd [21]). Oblique detonation waves may be retained stably during propagation through rectangular-cross-section bent tubes with a smaller inside wall area (or only by means of the linear motion of projectiles) than general planar detonation waves generated in tubes with a constant cross section.

Based on the result of numerical analysis, Lee et al. [22] indicated the effects of curvature on the detonation 
Proceedings of the Combustion Institute, Vol.33, 2011, pp.2319-2326

Y. Kudo, Y. Nagura, J. Kasahara, Y. Sasamoto, and A. Matsuo

wave propagation in annular channels and the critical curvature radius where the regular cell structure could be maintained. Deflagration-to-Detonation-Transition (DDT) occurring in the U-bent tubes has been experimentally revealed by Frolov et al. [23-24]. However, to our knowledge, no studies have reported steady-state oblique detonation waves stably propagating through the bent tubes.

The present study experimentally demonstrated that steady-state oblique detonation waves propagated stably through rectangular-cross-section bent tubes by visualizing the waves using a high-speed camera and the shadowgraph method. Moreover, the shapes of the oblique detonation waves propagating through the rectangular cross-section bent tubes and the critical curvature radiuses of the inside walls of the tubes were obtained.

The detonation diffracted from an open ended tube (Zel'dovich et al. [25], Matsui et al. [26], Mitrofanov and Soloukhin [27], Edward et al. [28], Knystautas et al. [29], Murry and Lee [30], Meredith et al. [31], M. Arienti, J. E. Shepherd [32]) is similar to that from bent tubes if an initial pressure of detonable mixture is sufficiently low, and inside-wall curvature radius is sufficiently large, but such diffracted detonation wave propagation is not always steady state. We focused on the stabilized detonation wave which could occur when the super critical condition was satisfied (an initial pressure of detonable mixture is sufficiently high, and inside-wall curvature radius is sufficiently small). This stabilized detonation wave is steady state in the two-dimensional polar coordinate in which the $\mathrm{z}$ (polar)-axis has the same rotating frequency as the detonation waves.

\section{Experiment}

Figs. 1 and 2 show the schematic of our experimental apparatus and the observation chamber, respectively. The observation chamber is composed of a rectangular cross-section bent tube 1, a circular cross-section straight tube 1, and a circular cross-section straight tube 2. As shown in Fig. 2, the circular cross-section straight tube 1 has a 50 $\mathrm{mm}$-length Shchelkin spiral and an igniter. The circular cross-section straight tube 2 is connected to a dump tank $\left(0.037 \mathrm{~m}^{3}\right)$ with a $12 \mu \mathrm{m}$ Mylar diaphragm between them. The inside and outside walls are fixed to the bold line area of the inside of the observation chamber (upper part in Fig. 2) to form a rectangular-cross-section bent tube with five kinds of curvature radiuses. In the upper part of Fig. 2, the rectangular-cross-section bent tube with an inside wall of $40 \mathrm{~mm}$ in curvature radius and an outside wall of $60 \mathrm{~mm}$ in curvature radius, which changes by 90 degrees in its axial direction, is shown. The rectangular-cross-section bent tubes are $20 \mathrm{~mm}$ in width and $16 \mathrm{~mm}$ in depth throughout their overall length.

The premixed gas filled in the observation chamber is ignited using a spark plug. Detonation waves are generated in the circular cross-section straight tube 1 with a $25.8 \mathrm{~mm}$ inside diameter and propagated into the rectangular-cross-section bent tube and then the circular cross-section straight tube 2 . High pressure gas generated by the detonation waves is discharged into the low-vacuum dump tank. The inside of the rectangular-cross-section bent tube is visualized using the shadowgraph method, as shown in Fig. 1. Shimadzu HPV-1 high-speed video camera was used.

Rectangular-cross-section bent tubes with 5, 10, 20, 40, or $60 \mathrm{~mm}$ in curvature radius $\left(r_{\mathrm{i}}\right)$ of the inside wall were used in our experiments, as shown in the lower part of Fig. 2. A stoichiometric ratio of ethylene-oxygen premixed gas was filled under an initial pressure $\left(p_{0}\right)$ of 20,30 , or $50 \mathrm{kPa}$. Table 1 summarizes the experimental conditions. We also carried out pressure measurements by using piezoelectric pressure sensors attached to the side channel wall and checked whether the velocity determined by the pressure was identical to the velocity obtained from the 
Proceedings of the Combustion Institute, Vol.33, 2011, pp.2319-2326

Y. Kudo, Y. Nagura, J. Kasahara, Y. Sasamoto, and A. Matsuo

high-speed camera. The results obtained showed that the velocity determined by the pressure was identical with that from the camera within measurement error limits of 2 to $14 \%$.

\section{Oblique-Detonation-Wave Shape}

The geometrical shapes of steady-state oblique detonation waves, which propagate stably through the rectangular-cross-section bent tubes, were calculated. As shown in Fig. 3, the center of the curvature radius of the inside and outside walls is assumed to be an origin $\mathrm{O}$. The oblique detonation fronts $(r, \theta)$ are estimated by means of two-dimensional polar coordinates. It is assumed that the detonation waves propagate along the inside wall surface. Assuming that an angle defined by a line extending in the $\theta$ direction and the detonation wave at a point $(r, \theta)$ is $\phi$ and the normal propagation velocity of the oblique detonation wave is $V_{\mathrm{CJ}}$ on all detonation fronts, the following relationship can be established on the fronts $(r, \theta)$.

$$
\begin{aligned}
& \sin \phi=\frac{V_{\mathrm{CJ}}}{r \omega_{\mathrm{i}}} \\
& \tan \phi=-\frac{\mathrm{d} r}{r \mathrm{~d} \theta}
\end{aligned}
$$

Where, $r_{\mathrm{i}} \omega_{1}=V_{\mathrm{CJ}}\left(\phi_{1}=\pi / 2\right)$. If we observe these stabilized detonation waves in the bent tubes from the two-dimensional polar coordinate in which the $\mathrm{z}$ (polar)-axis has the same rotating frequency as the detonation waves, the detonation wave propagates in steady state. Since the detonation wave reflects at the inside wall, the wave must be perpendicular to the wall or a reflection wave will occur. In the present experiment there was no reflection wave in the vicinity of the inside wall, and we therefore assumed the detonation wave was perpendicular to the inside wall. The following differential equation is derived from Eq.(1) and Eq.(2).

$$
-\frac{\mathrm{d} r}{r \mathrm{~d} \theta}=\frac{V_{\mathrm{CJ}}}{\sqrt{r^{2} \omega_{\mathrm{i}}^{2}-V_{\mathrm{CJ}}^{2}}}
$$

Assuming that $\theta$ is $\theta_{\mathrm{i}}$ with $r=r_{\mathrm{i}}$, the integration of Eq. (3) gives the following equation.

$$
\theta-\theta_{\mathrm{i}}=-\frac{\sqrt{r^{2} \omega_{\mathrm{i}}^{2}-V_{\mathrm{CJ}}^{2}}}{V_{\mathrm{CJ}}}+\tan ^{-1}\left(\frac{\sqrt{r^{2} \omega_{\mathrm{i}}^{2}-V_{\mathrm{CJ}}^{2}}}{V_{\mathrm{CJ}}}\right)
$$

Assuming that $\mathrm{R}=r \omega_{1} / V_{\mathrm{CJ}}=r / r_{\mathrm{i}}$, the following Eq. (5) is obtained.

$$
\theta-\theta_{\mathrm{i}}=-\sqrt{\mathrm{R}^{2}-1}+\tan ^{-1} \sqrt{\mathrm{R}^{2}-1}
$$

Fig. 4 shows the relationship between $\theta-\theta_{\mathrm{i}}$ obtained by Eq. (5) and R.

\section{Results and Discussions}

The results of the visualization experiments are shown in Fig. 5 as overlapped detonation fronts. The detonation cell width is about $0.8 \mathrm{~mm}(50 \mathrm{kPa})$. As the exposure time of the high-speed camera was $250 \mathrm{~ns}$, the spatial resolution in the wave-propagation direction is about $0.5 \mathrm{~mm}$. By this low spatial resolution of the camera, we were not able to capture the detonation cellular structures.

The steady-state oblique detonation waves $(\omega=\dot{\theta}=$ const.) [Fig.5, shot No. 9, 11, 12, 14, 15], which propagate 
Proceedings of the Combustion Institute, Vol.33, 2011, pp.2319-2326

Y. Kudo, Y. Nagura, J. Kasahara, Y. Sasamoto, and A. Matsuo

stably through the tubes, were generated with high $p_{0}$ values and large $r_{\mathrm{i}}$ values, while unsteady-state oblique detonation waves accompanied by diffraction and re-initiation [Fig. 5, shot No. 1-8, 10, 13] were generated with low $p_{0}$ values and small $r_{\mathrm{i}}$ values. The latter detonation waves were diffracted on the inside wall of the tube. The reaction area of the detonation wave was separated from that of the shock waves and the transverse detonation wave started to propagate at a re-initiation point.

Fig. 6 shows a change in the shape of a detonation front under the conditions, $p_{0}=50 \mathrm{kPa}, r_{\mathrm{i}}=20 \mathrm{~mm}$ (shot No. 9). In Fig. 6, $r$ was plotted against $\theta-\theta_{\mathrm{i}}$. As shown in Fig. 3, $\theta_{\mathrm{i}}$ is the circumferential angle of a detonation front on the inside wall. As shown in Fig. 6, the shape of the detonation wave is formed asymptomatically into a fixed shape at $\theta_{\mathrm{i}}=90^{\circ}$. In shots No. $9,11,12,14$, and 15 , the shape of the detonation wave is almost fixed at $\theta_{\mathrm{i}}=90^{\circ}$. Specifically, the shape of the detonation wave was fixed when the propagation direction of the detonation wave changed by $90^{\circ}$ on the inside wall of the tube.

Fig. 7 shows the comparison between the shapes of the steady-state oblique detonation waves, which propagate stably through the tubes $\left[p_{0}=50 \mathrm{kPa}, r_{\mathrm{i}}=20,40,60 \mathrm{~mm}\right.$ (shot No. 9, 12, 15)], and those calculated by Eq. (4). Fig. 8 shows the angular velocity $\omega$ of the wave front (shot No. 9). As shown in Fig. 8, on the fronts of the oblique detonation waves, $\omega$ approaches $100000 \pm 5000 \mathrm{rad} / \mathrm{s}$ asymptotically. The $\omega$ values in the other shots are listed in Table 1. In Figs. 9-11, the front velocity $r_{\mathrm{i}} \omega$ of the detonation wave with $r=r_{\mathrm{i}}$ is plotted against $\theta$. Some front velocity, which always satisfies a relational expression $r_{\mathrm{i}} \omega \geq 0.8 V_{\mathrm{CJ}}$, is marked with a black circle, indicating a stabilized condition in Fig. 12. Under the stabilized condition, $r_{\mathrm{i}}$ was 20,40 , or $60 \mathrm{~mm}$ under $p_{0}=50 \mathrm{kPa}$ and 40 or $60 \mathrm{~mm}$ under $p_{0}=30 \mathrm{kPa}$. Any front velocity, which always satisfies a relational expression $0.6 V_{\mathrm{CJ}} \leq r_{\mathrm{i}} \omega<$ $0.8 V_{\mathrm{CJ}}$, is marked with a black triangle, indicating a critical condition in Fig. 12. Front velocity, which satisfies a relational expression $r_{\mathrm{i}} \omega<0.6 V_{\mathrm{CJ}}$, is marked with a cross, indicating a not-stabilized condition in Fig. 12. Under the not-stabilized condition (an initial pressure of detonable mixture is sufficiently low, and inside-wall curvature radius is sufficiently large), decoupled shock-induced combustion occurred. Periodically and alternately, the decoupled shock-induced combustion and the detonation wave appeared in the vicinity of the inside wall of the bent tube. The phenomena were always in an unsteady state in any coordinate. These phenomena can be explained by the detonation diffraction studied well by many other researchers [25-32]."

In Fig. $13, r_{\mathrm{i}} / \lambda$ is plotted against $p_{0}$ where $\lambda$ indicates the detonation cell width. $72.312 p_{0}^{-1.1362}\left(\lambda[\mathrm{mm}], p_{0}\right.$ $[\mathrm{kPa}]$ ) was extracted from the Detonation Database (Kaneshige and Shepherd [33], Knystautas et al. [29], Abid et al. [34], Strehlow [35]) of the California Institute of Technology to use as $\lambda$.

From Fig. 13, the critical-condition detonation waves were observed in the range $17 \leqq r_{\mathrm{i}} / \lambda \leqq 26$, and the critical curvature radius may exist in the range $14 \leqq r_{\mathrm{i}} / \lambda \leqq 40$.

Self-sustained oblique detonation waves are usually stabilized by an over driven detonation generated in the vicinity of the blunt-nosed projectile [14-21] or by the interaction between an oblique shock wave and a reaction wave on the wedge ( $\mathrm{Li}$ et al. [36]). In the present experiment, however, the self-sustained oblique detonation wave was stabilized by the curved inside wall to which the detonation wave was perpendicular. Under such a curved inside-wall stabilized condition, the detonation wave has a small curvature (divergence), but maintains a steady state.

The critical conditions for stabilizing the detonation wave can be discussed by the Yao-and-Stewart velocity-curvature relation for detonation waves. Yao and Stewart [37] obtained the master equation by assuming that a detonation wave is quasi-steady and quasi-one-dimensional, and that the detonable gas has Arrenius kinetics and large activation energy. We 
Proceedings of the Combustion Institute, Vol.33, 2011, pp.2319-2326

Y. Kudo, Y. Nagura, J. Kasahara, Y. Sasamoto, and A. Matsuo

calculated the critical curvature radius $r_{\mathrm{cr}}=1 / \kappa_{\mathrm{cr}}=2.6 l_{\mathrm{c}}$ for $E=4.2[\mathrm{MJ} / \mathrm{kg}], \gamma=1.2, D_{\mathrm{CJ}}=2343[\mathrm{~m} / \mathrm{s}]$ by Equation 25 in Ref. 37, where $l_{\mathrm{c}}$ is the induction zone length scale and $E$ is the activation energy. From this calculation, we found the critical curvature radiuses of the quasi-one-dimensional detonation waves were very small and comparable to the induction length scales, which were much smaller than the cell width in our experimental conditions. This calculated critical curvature radius is quantitatively different from our experimental detonation-wave critical scale, $14 \lambda \leqq r_{\mathrm{i}} \leqq 40 \lambda$ (critical inside wall radius); however, we think that qualitatively the curvature-propagation-velocity relationship and critical condition for stabilization can be discussed by Yao and Stewart analysis. An actual detonation wave front has a three-dimensional structure and the front is always unstable. This actual unstable three-dimensional detonation front diverges more strongly than the quasi-one-dimensional model's detonation front. This difference can explain the disagreement in the critical curvatures between the experiment and the model.

\section{Conclusions}

The present study has experimentally demonstrated that steady-state oblique detonation waves propagated stably through rectangular-cross-section bent tubes by visualizing these waves using a high-speed camera and the shadowgraph method. The shapes of the oblique detonation waves propagating through the rectangular cross-section bent tubes and the critical curvature radiuses of the inside walls of these tubes were examined. The oblique detonation waves were stabilized under the conditions of high initial pressure and a large curvature radius of the inside wall of the rectangular-cross-section bent tube. The geometrical shapes of the stabilized detonation waves were calculated, and were in good agreement with those obtained from our experiments. Moreover, it was experimentally shown that the critical condition under which steady-state oblique detonation waves could stably propagate through the rectangular-cross-section bent tubes was the curvature radius of the inside wall of the rectangular-cross-section bent tube equivalent to 14 to 40 times the cell width.

\section{Acknowledgements}

This work was subsidized by the Ministry of Education, Culture, Sports, Science and Technology, Japan, a Grant-in-Aid for Scientific Research (A), No.20241040; a Grant-in-Aid for Scientific Research (B), No. 21360411, and by the Research Grant Program from the Institute of Space and Astronautical Science, the Japan Aerospace Exploration Agency.

\section{References}

[1] K. Kailasanath, AIAA J. 38 (9) (2000) 1698-1708.

[2] K. Kailasanath, AIAA J. 41 (2) (2003) 145-159.

[3] G.D. Roy, S.M. Frolov, A.A. Borisov, D.W. Netzer, Progress in Energy and Combustion Science 30 (6) (2004) 545-672.

[4] J. Kasahara, M. Hirano, A. Matsuo, Y. Daimon, T. Endo, Journal of Propulsion and Power 25 (6) (2009) 1281-1290.

[5] J. Kasahara, A. Hasegawa, T. Nemoto, H. Yamaguchi, T. Yajima, T. Kojima, Journal of Propulsion and Power 25 (1) (2009) 173-180.

[6] J. Kasahara, Z. Liang, S.T. Browne, J.E. Shepherd, AIAA J. 46 (7) (2008) 1593-1603. 
Proceedings of the Combustion Institute, Vol.33, 2011, pp.2319-2326

Y. Kudo, Y. Nagura, J. Kasahara, Y. Sasamoto, and A. Matsuo

[7] S. Sato, A. Matsuo, T. Endo, J. Kasahara, Journal of Propulsion and Power 22 (1) (2006) 64-69.

[8] T. Endo, J. Kasahara, A. Matsuo, K. Inaba, S. Sato, T. Fujiwara, AIAA Journal 42 (9) (2004) 1921-1929.

[9] T. Endo, T. Yatsufusa, S. Taki, A. Matsuo, K. Inaba, J. Kasahara, Journal of Propulsion and Power 23 (5) (2007) 1033-1041.

[10] J. Meredith, H.D. Ng, J.H.S. Lee, Detonation Diffraction from an Annular Channel, 22nd ICDERS (2009).

[11] J. Kindracki, P. Wolanski, Z. Gut, Experimental Research on Rotating Detonation in Gaseous Fuels-Oxygen Mixtures, 22nd ICDERS (2009).

[12] X.H. Jiang, B.C. Fan, M.Y. Gui, Z.H. Chen, Numerical Investigations on the Three -dimensional Flow Patterns of the Continuous Rotation Detonation, 22nd ICDERS (2009).

[13] T.H. Yi, J. Lou, P. Wolanski, C. Turangan, B.C. Khoo, Propulsive Performance Study of Rotational Detonation Engine, 22nd ICDERS (2009).

[14] J. Kasahara, T. Arai, S. Chiba, K.Takazawa, Y. Tanahashi, A.Matsuo, Proc. Combust. Inst. 29 (2002) 2817-2824.

[15] J. Kasahara, T. Horii, T. Endo, T. Fujiwara, Proc. Combust. Inst. 26 (1996) 2903-2908.

[16] J. Kasahara, T. Fujiwara, T. Endo, T. Arai, AIAA J.39 (2001) 1553-1561.

[17] J. Kasahara, T. Arai, S. Chiba, A. Matsuo, Proc. Jpn. Symp. Combust. 39 (2001) 451-452.

[18] A.J. Higgins, AIAA Paper 97-3179. (1997).

[19] A.J. Higgins, Investigation of Detonation Initiation by Supersonic Blunt Bodies, Ph.D. dissertation, University of Washington, Seattle, 1996.

[20] J.H.S. Lee, Prog. Astronaut. Aeronaut. 173 (1997) 293-310.

[21] M.J. Kaneshige, J.E. Shepherd, Proc. Combust. Inst. 26 (1996) 3015-3022.

[22] S.H. Lee, D.R. Jo, J.Y. Choi, AIAA Paper 2008-988. (2008)

[23] S.M. Frolov, V.S. Aksenov, I.O. Shamshin, Proc. Combust. Inst. 31 (2006) 2421-2428.

[24] S.M. Frolov, V.S. Aksenov, Dokalady Physical Chemistry 416 (1) (2007) 261-264.

[25] Ya.B. Zeldovich, S.M. Kogarko, N.N. Simonov, Sov. Phys. Tech. Phys. 1 (1956) 1689-1713.

[26] H. Matsui, J.H.S. Lee, Proc. Combust. Inst. 17 (1979) 1269-1280.

[27] V.V. Mitrofanov, R.I. Soloukhin, Sov. Phys. Doklady 9 (1965) 1055.

[28] H. Edward, G.O. Thomas, M.A. Nettleton, J. Fluid Mech. 95 (1979) 95-79.

[29] R. Knystautas, J.H.S. Lee, C. Guirao, Combust. Flame 48 (1982) 63-83.

[30] S.B. Murry, J.H.S. Lee, Combust. Flame 52 (1983) 269-289.

[31] J. Meredith, D.Ng. Hoi, J.H.S. Lee., 22nd ICDERS (2009).

[32] M. Arienti, J. E. Shepherd, J. Fluid Mech. 529 (2005) 117-147.

[33] M. Kaneshige and J.E. Shepherd, Detonation database. Technical Report FM97-8, GALCIT, July 1997. available at http://www.galcit.caltech.edu/detn_db/html/db.html

[34] S. Abid, G. Dupre, C. Paillard, Prog. Astronaut. Aeronaut. 153 (1991) 162-181.

[35] R.A. Strehlow, AIAA J. 7 (3) (1969) 492-496. 
Proceedings of the Combustion Institute, Vol.33, 2011, pp.2319-2326

Y. Kudo, Y. Nagura, J. Kasahara, Y. Sasamoto, and A. Matsuo

[36] C. Li, K. Kailasanath, E.S. Oran, Physics Fluids 6 (1994) 1600-1611.

[37] J. Yao, D.S. Stewart, Combust. Flame 100 (1995) 519-528. 


\section{Figure captions}

Table 1 Experimental Conditions

Fig. 1. Experimental apparatus

Fig. 2. Observation chamber, inside and outside walls

Fig. 3. Oblique-detonation-wave shape defined by a polar coordinate

Fig. 4. Ideal shape of an oblique detonation wave in a rectangular cross-section tube

Fig. 5. Fig. 5. Experimental results of overlapped detonation fronts. Steady-state stabilized oblique detonation waves [shot No. 9, 11, 12, 14, 15], and unsteady-state oblique detonation waves [shot No. 1-8, 10, 13], All inter-frame time $=2 \mu \mathrm{s}$

Fig. 6. Transition from a planar detonation wave to an oblique detonation wave in a rectangular cross-section tube $\left[p_{0}=50 \mathrm{kPa}, r_{\mathrm{i}}=20 \mathrm{~mm}\right.$ (shot. No. 9)]

Fig. 7. Comparison of experimental oblique detonation shape with calculated shape from Eq. (4) [shot No. $9,12,15], V_{\mathrm{CJ}}=2342 \mathrm{~m} / \mathrm{s}$

Fig. 8. Angular velocity plotted against wave angle $\left[p_{0}=50 \mathrm{kPa}, r_{\mathrm{i}}=20 \mathrm{~mm}\right.$ (shot. No. 9)]

Fig. 9. Wave propagation velocity on the inside wall plotted against wave angle $\left[p_{0}=50 \mathrm{kPa}\right.$ (shot No. $3,6,9,12,15)$

Fig. 10. Wave propagation velocity on the inside wall plotted against wave angle $\left[p_{0}=30 \mathrm{kPa}\right.$ (shot No. $2,5,8,11,14)$

Fig. 11. Wave propagation velocity on the inside wall plotted against wave angle $\left[p_{0}=20 \mathrm{kPa}\right.$ (shot No. $1,4,7,10,13)$

Fig. 12. Critical conditions for stabilized oblique detonation in a rectangular cross-section tube

Fig. 13. Critical conditions for stabilized oblique detonation in a rectangular cross-section tube by $r_{\mathrm{i}} / \lambda$ 
Proceedings of the Combustion Institute, Vol.33, 2011, pp.2319-2326

Y. Kudo, Y. Nagura, J. Kasahara, Y. Sasamoto, and A. Matsuo

Table 1. Experimental Conditions

\begin{tabular}{cccc}
\hline $\begin{array}{c}\text { shot } \\
\text { No. }\end{array}$ & $r_{\mathrm{i}}$ & $p_{0}$ & $\omega$ \\
\hline & {$[\mathrm{mm}]$} & {$[\mathrm{kPa}]$} & {$[\mathrm{rad} / \mathrm{s}]$} \\
\hline 1 & 5 & $20 \pm 1$ & - \\
2 & 5 & $30 \pm 1$ & - \\
3 & 5 & $50 \pm 1$ & - \\
4 & 10 & $20 \pm 1$ & - \\
5 & 10 & $30 \pm 1$ & - \\
6 & 10 & $50 \pm 1$ & - \\
7 & 20 & $20 \pm 1$ & - \\
8 & 20 & $30 \pm 1$ & - \\
9 & 20 & $50 \pm 1$ & $100000 \pm 5000$ \\
10 & 40 & $20 \pm 1$ & - \\
11 & 40 & $30 \pm 1$ & $51000 \pm 4000$ \\
12 & 40 & $50 \pm 1$ & $55000 \pm 3000$ \\
13 & 60 & $20 \pm 1$ & - \\
14 & 60 & $30 \pm 1$ & $35000 \pm 3000$ \\
15 & 60 & $50 \pm 1$ & $37500 \pm 3000$ \\
\hline & & &
\end{tabular}

(single column 256 words) 
Proceedings of the Combustion Institute, Vol.33, 2011, pp.2319-2326

Y. Kudo, Y. Nagura, J. Kasahara, Y. Sasamoto, and A. Matsuo

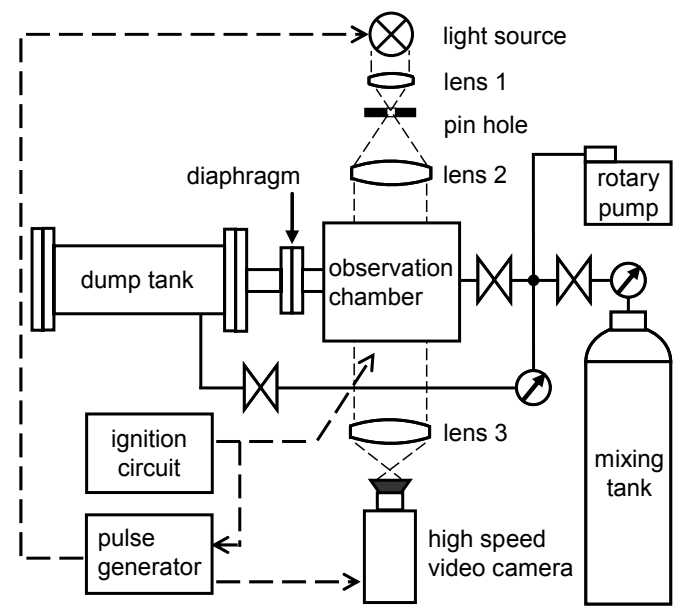

Fig. 1. Experimental apparatus

(single column 160 words) 
Proceedings of the Combustion Institute, Vol.33, 2011, pp.2319-2326

Y. Kudo, Y. Nagura, J. Kasahara, Y. Sasamoto, and A. Matsuo

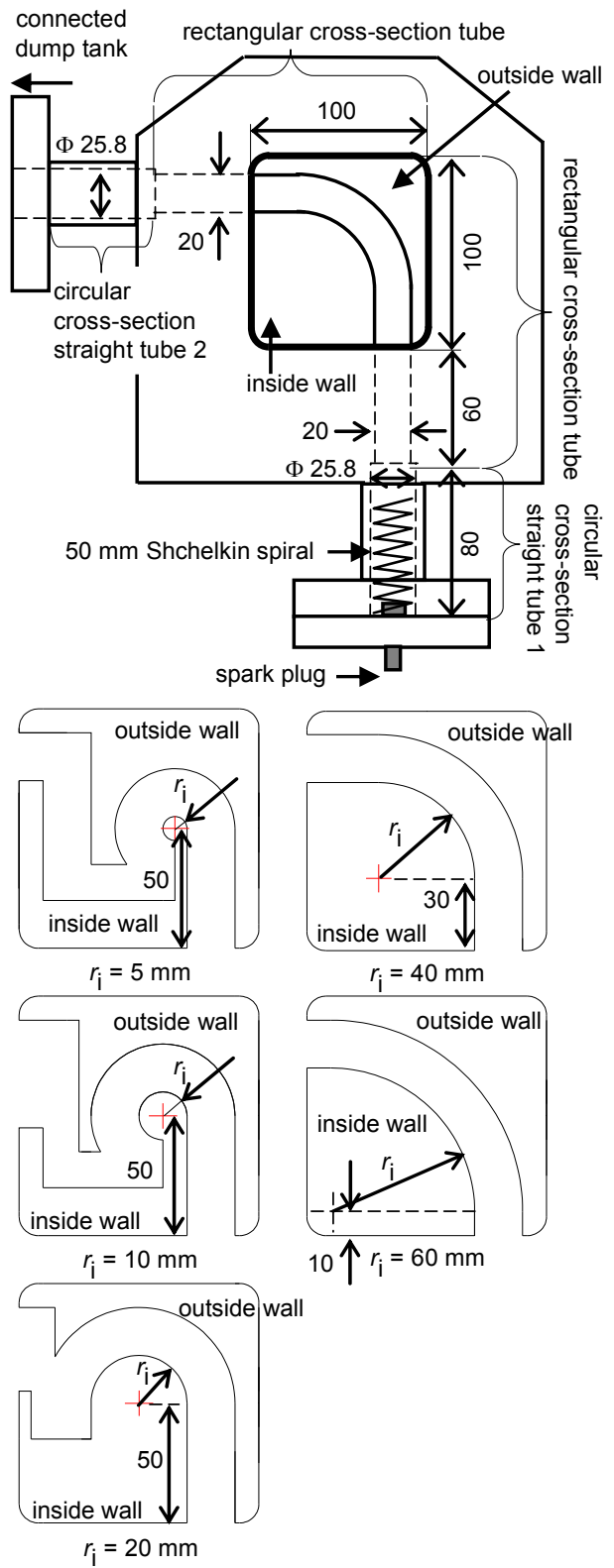

Fig. 2. Observation chamber, inside and outside walls (single column 384 words) 
Proceedings of the Combustion Institute, Vol.33, 2011, pp.2319-2326

Y. Kudo, Y. Nagura, J. Kasahara, Y. Sasamoto, and A. Matsuo

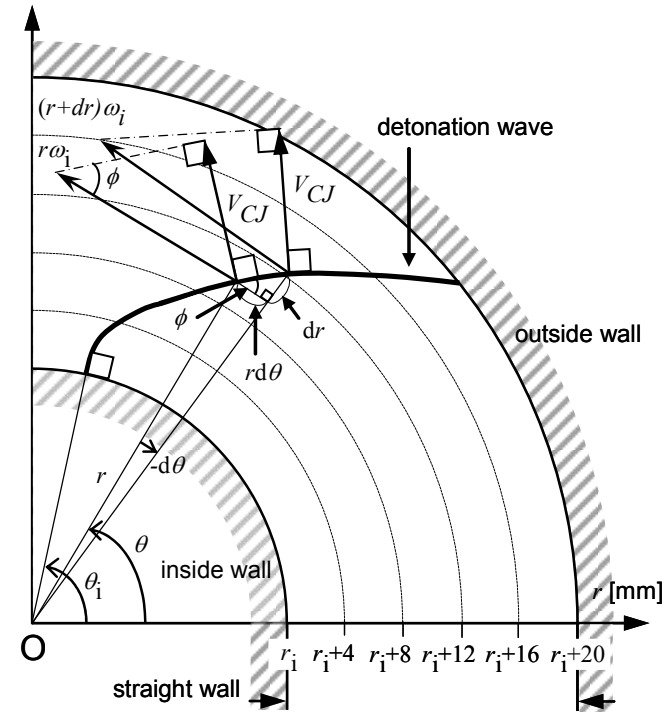

Fig. 3. Oblique-detonation-wave shape defined by a polar coordinate (single column 193 words) 
Proceedings of the Combustion Institute, Vol.33, 2011, pp.2319-2326

Y. Kudo, Y. Nagura, J. Kasahara, Y. Sasamoto, and A. Matsuo

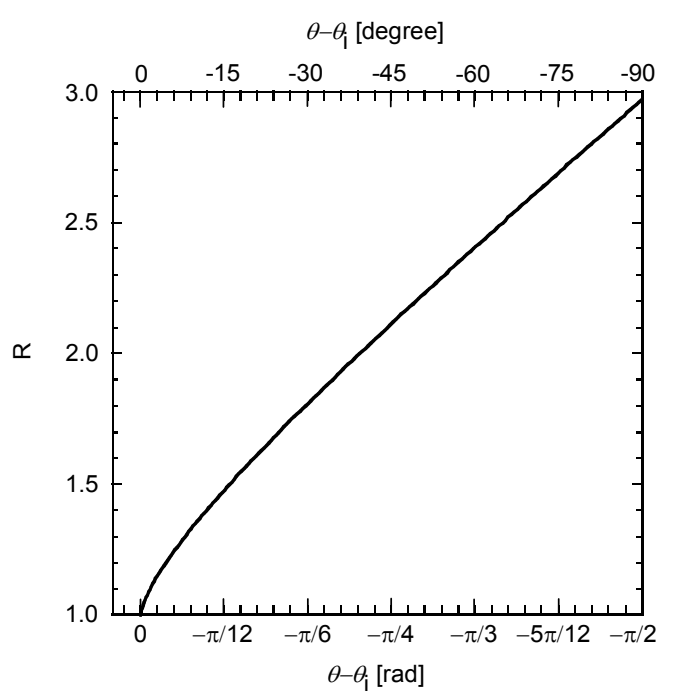

Fig. 4. Ideal shape of an oblique detonation wave in a rectangular cross-section tube (single column 190 words $(=((70+10) * 2.2)+14))$ 
Proceedings of the Combustion Institute, Vol.33, 2011, pp.2319-2326

Y. Kudo, Y. Nagura, J. Kasahara, Y. Sasamoto, and A. Matsuo

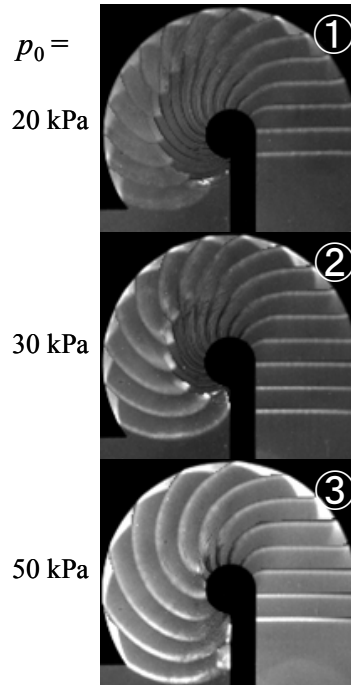

$r_{\mathrm{i}}=5 \mathrm{~mm}$

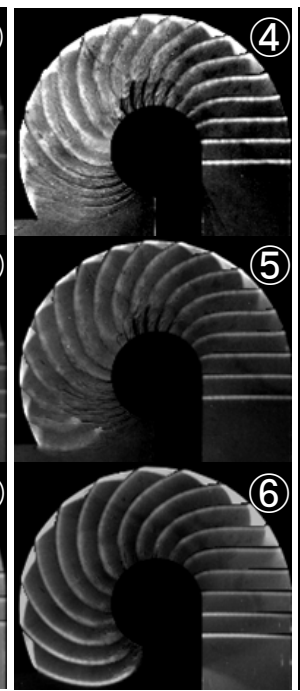

$10 \mathrm{~mm}$

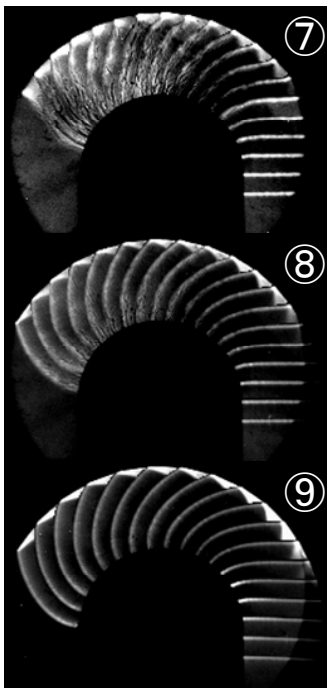

$20 \mathrm{~mm}$

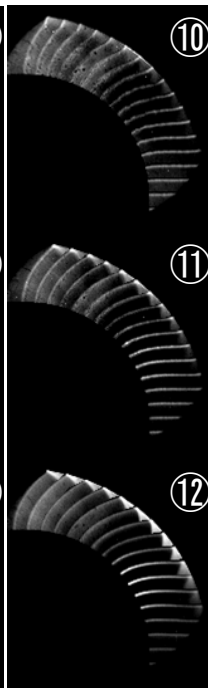

$40 \mathrm{~mm}$

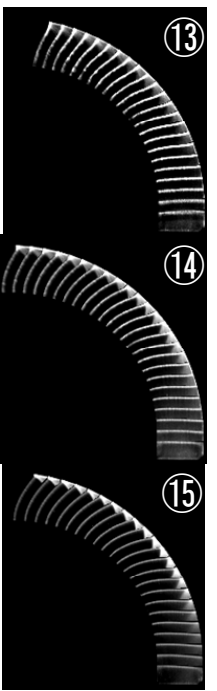

$60 \mathrm{~mm}$

Fig. 5. Experimental results of over lapped detonation fronts. Steady-state stabilized oblique detonation waves [shot No. 9, 11, 12, 14, 15], and unsteady-state oblique detonation waves [shot No. 1-8, 10, 13]; All inter-frame time $=2 \mu \mathrm{s}$

(double column 424 words $(((78+10) * 4.4)+36))$ 
Proceedings of the Combustion Institute, Vol.33, 2011, pp.2319-2326

Y. Kudo, Y. Nagura, J. Kasahara, Y. Sasamoto, and A. Matsuo

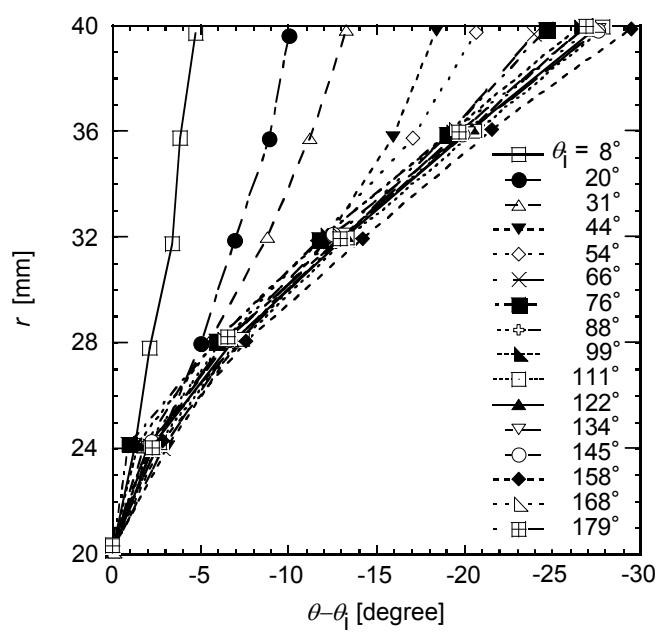

Fig. 6. Transition from a planar detonation wave to an oblique detonation wave in a rectangular cross-section tube

$$
\left[p_{0}=50 \mathrm{kPa}, r_{\mathrm{i}}=20 \mathrm{~mm}\right. \text { (shot. No. 9)] }
$$

(single column 187 words) 
Proceedings of the Combustion Institute, Vol.33, 2011, pp.2319-2326

Y. Kudo, Y. Nagura, J. Kasahara, Y. Sasamoto, and A. Matsuo

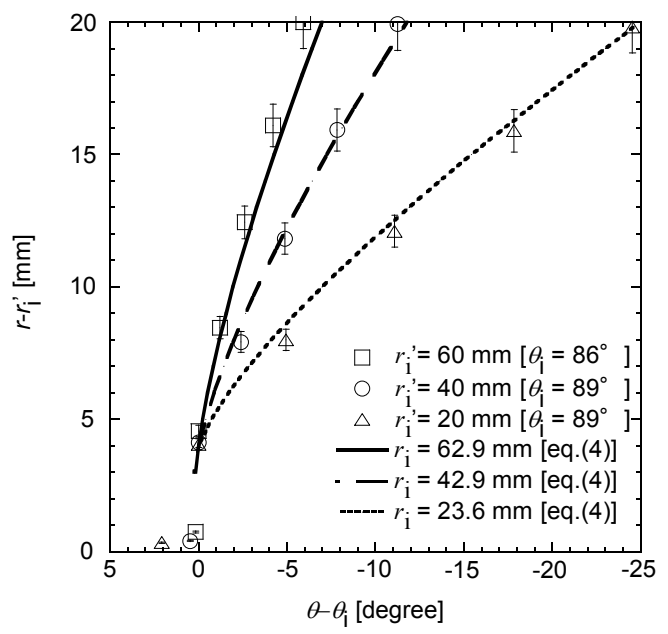

Fig. 7. Comparison of experimental oblique detonation shape with calculated shape from Eq. (4) [shot No. 9, 12, $15], V_{\mathrm{CJ}}=2342 \mathrm{~m} / \mathrm{s}$

(single column 180 words) 
Proceedings of the Combustion Institute, Vol.33, 2011, pp.2319-2326

Y. Kudo, Y. Nagura, J. Kasahara, Y. Sasamoto, and A. Matsuo

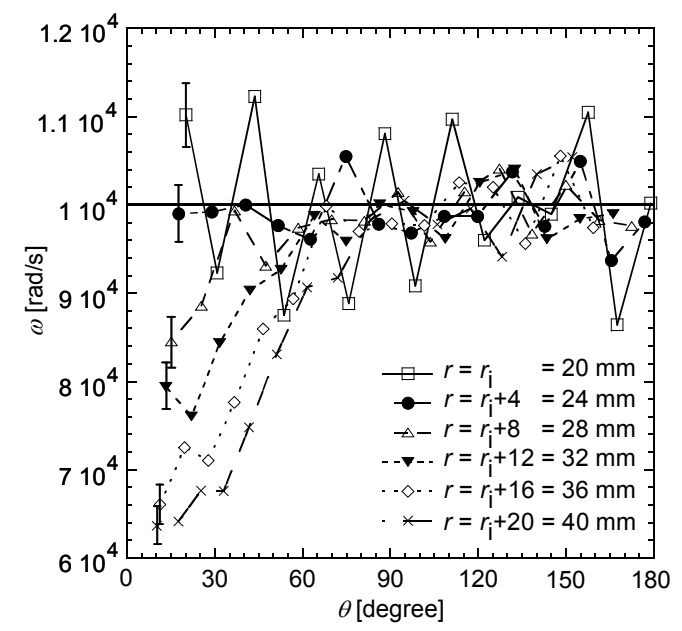

Fig. 8. Angular velocity plotted against the wave angle $\left[p_{0}=50 \mathrm{kPa}, r_{\mathrm{i}}=20 \mathrm{~mm}\right.$ (shot. No. 9)] (single column 176 words) 
Proceedings of the Combustion Institute, Vol.33, 2011, pp.2319-2326

Y. Kudo, Y. Nagura, J. Kasahara, Y. Sasamoto, and A. Matsuo

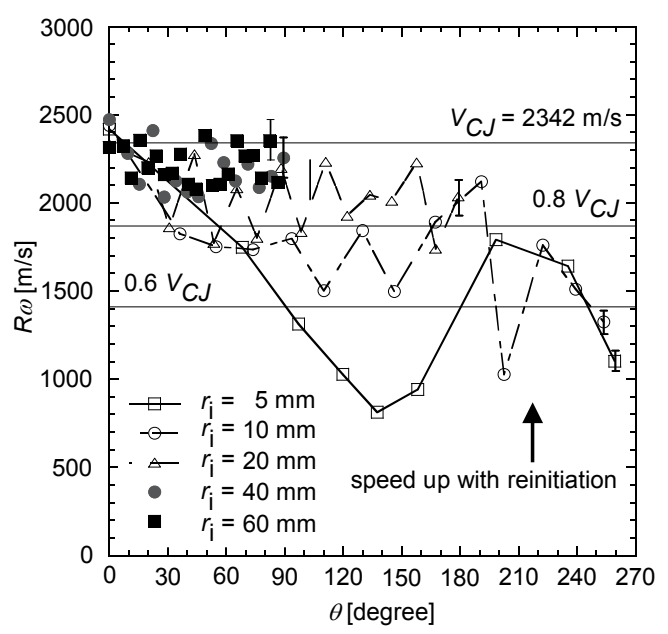

Fig. 9. Wave propagation velocity on the inside wall plotted against the wave angle $\left[p_{0}=50 \mathrm{kPa}\right.$ (shot No. $3,6,9$, $12,15)$

(single column 182 words) 
Proceedings of the Combustion Institute, Vol.33, 2011, pp.2319-2326

Y. Kudo, Y. Nagura, J. Kasahara, Y. Sasamoto, and A. Matsuo

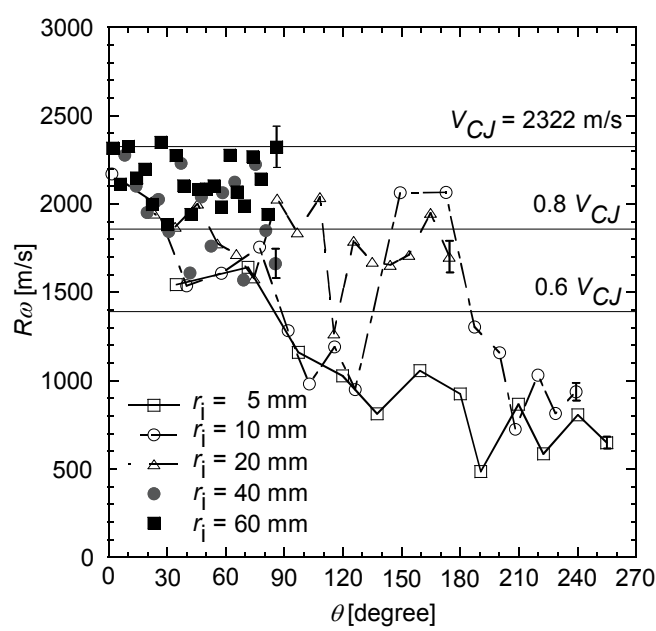

Fig. 10. Wave propagation velocity on the inside wall plotted against the wave angle $\left[p_{0}=30 \mathrm{kPa}\right.$ (shot No. $2,5,8$, $11,14)$

(single column 182 words) 
Proceedings of the Combustion Institute, Vol.33, 2011, pp.2319-2326

Y. Kudo, Y. Nagura, J. Kasahara, Y. Sasamoto, and A. Matsuo

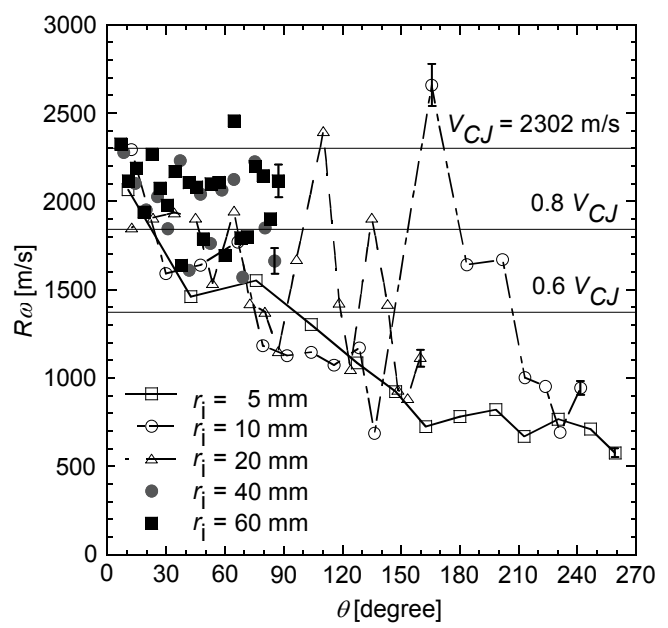

Fig. 11. Wave propagation velocity on the inside wall plotted against the wave angle $\left[p_{0}=20 \mathrm{kPa}\right.$ (shot No. $1,4,7$, $10,13)$

(single column 182 words) 
Proceedings of the Combustion Institute, Vol.33, 2011, pp.2319-2326

Y. Kudo, Y. Nagura, J. Kasahara, Y. Sasamoto, and A. Matsuo

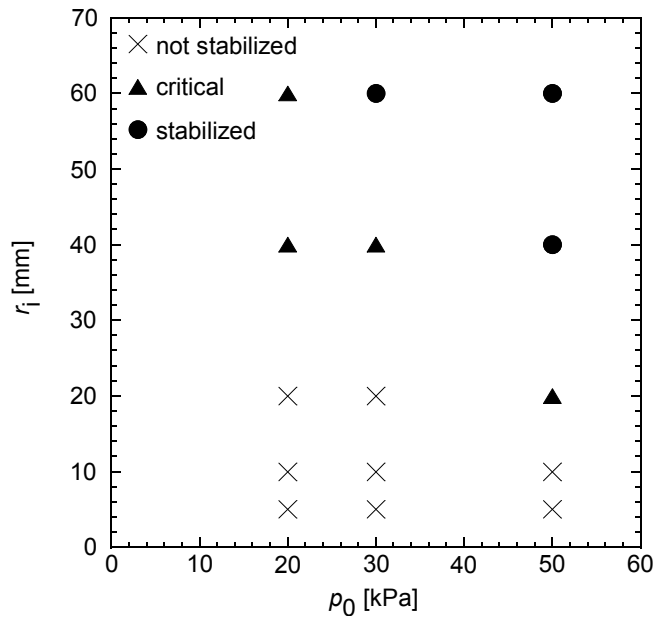

Fig. 12. Critical conditions for stabilized oblique detonation in a rectangular cross-section tube (single column 173 words) 
Proceedings of the Combustion Institute, Vol.33, 2011, pp.2319-2326

Y. Kudo, Y. Nagura, J. Kasahara, Y. Sasamoto, and A. Matsuo

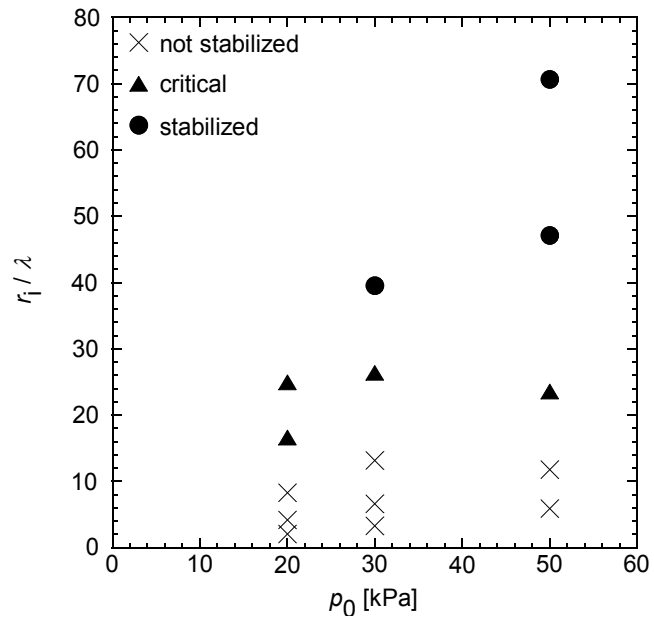

Fig. 13. Critical conditions for stabilized oblique detonation in a rectangular cross-section tube by $r_{\mathrm{i}} / \lambda$ (single column 176 words) 